\title{
Broadband Study of Inter-modal Bragg Scattering Four Wave Mixing in Multi-Mode Fibres
}

\author{
O. F. Anjum (1), P. Horak(1), Y. Jung ${ }^{(1)}$, M. Suzuki(2), Y. Yamamoto(2), T. Hasegawa(2), P. Petropoulos ${ }^{(1)}$ \\ D. J. Richardson ${ }^{(1)}$, F. Parmigiani ${ }^{(1)}$ \\ (1) Optoelectronics Research Centre, University of Southampton, U. K., frp@orc.soton.ac.uk \\ (2) Optical Communications Laboratory, Sumitomo Electric Industries, Ltd. Japan.
}

\begin{abstract}
We report on the design, fabrication and experimental characterization of Germanium-doped graded-index multi-mode fibres to achieve broadband operation for the inter-modal Bragg scattering four wave mixing process.
\end{abstract}

\section{Introduction}

Multi-mode nonlinear media offer an additional (spatial) degree of freedom to the manipulation of signals as compared to those that support just a single-mode, and could therefore constitute an interesting means of enhancing the performance of many ultra-fast signal processing applications ${ }^{15}$. This potential, in combination with the tremendous developments in space division multiplexing, advanced fibre fabrication, and the ability to independently launch and control highorder modes (HOMs), has triggered a recent revival of interest in nonlinear effects in multimode fibres (MMFs).

Among the various types of signal-agnostic nonlinear effects, the Bragg scattering (BS) four wave mixing (FWM) process generates a copy of the signal ideally without adding noise ${ }^{6}$. The new frequency of the idler is shifted from the original by as much as the frequency spacing between two stronger waves (typically referred to as the pumps). In single-mode systems, this pump-topump frequency spacing $\left(\Delta \omega_{\mathrm{pp}}\right)$ is typically only of the order of hundreds of gigahertz due to the specific fibre dispersion profiles. Using a $1 \mathrm{~km}$ long three-mode fibre we previously demonstrated intermodal FWM processes in multiple distinct bands (within the C-band or between the C- and L-bands), by simply exciting different HOMs of the same fibre ${ }^{1-2}$. For the intermodal BS FWM case, we have already shown the potential to only phase-match a specific nonlinear process, the BS red-shifted (BSr) one ${ }^{2}$. This is a key feature of optical processors for inclusion in telecommunication networks, as any other undesired nonlinear effects may degrade the overall system performance. So far, we have demonstrated this possibility only for relatively small $\Delta \omega_{\mathrm{pp}}$ bandwidths of about $1 \mathrm{~nm}^{2}$.

Here, we report on the design, fabrication and experimental characterization of a class of dispersion-tailored Ge-doped graded-index (GI) MMFs. The fibres were engineered to achieve large $\Delta \omega_{\mathrm{pp}}$ bandwidths for the phase-matched BSr FWM process when the pump waves excite the fundamental mode $\left(\mathrm{LP}_{01}\right)$, while the signal and corresponding generated idlers are in the first HOM (LP 11$)$, respectively. For the fabricated GI MMFs, simulations predict $\Delta \omega_{\mathrm{pp}}$ of almost 18 $\mathrm{nm}$ at the $-3 \mathrm{~dB}$ half-bandwidth and experimentally we demonstrated up to $7 \mathrm{~nm}$ for a $\Delta \omega_{\text {pump-signal }}$ of $22.6 \mathrm{~nm}$.

\section{Fibre design and fabrication}

For the arrangement of the pumps, signal and BS blue- and red-shifted (BSb and BSr) idlers as shown in Fig. 1(a), broadband phase matching is achieved when the inverse group velocity (IGV) curves of the two modes are exact shifted replicas of one another ${ }^{1-2}$, when small pump-topump wavelength detunings are assumed. We were able to obtain this in the bands of interest (C-band) for the $\mathrm{LP}_{01}$ and $\mathrm{LP}_{11}$ modes by designing GI MMFs with the truncated parabolic
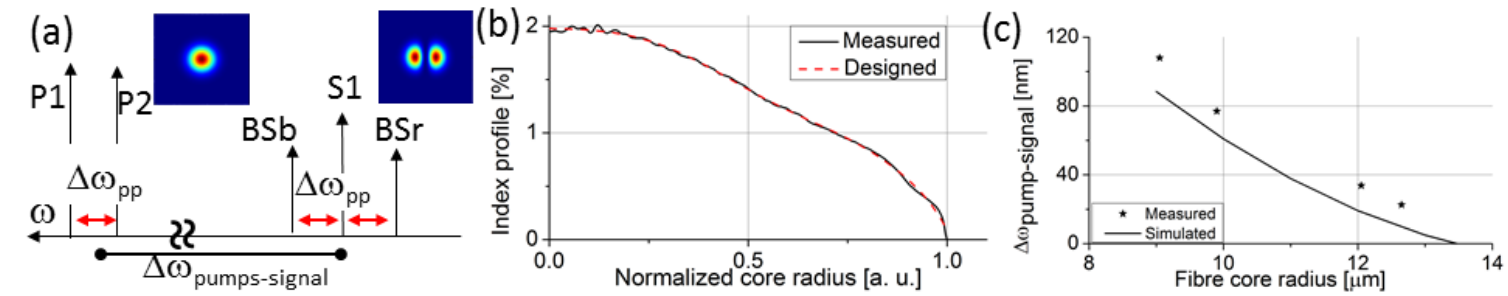

Fig. 1: (a) Wave and wavelength allocation. (b) Simulated and measured index profile of the GI MMF. (c) Simulated (line)

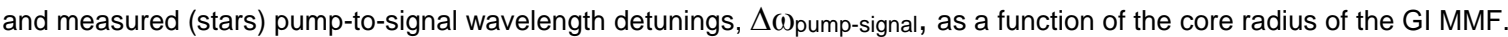



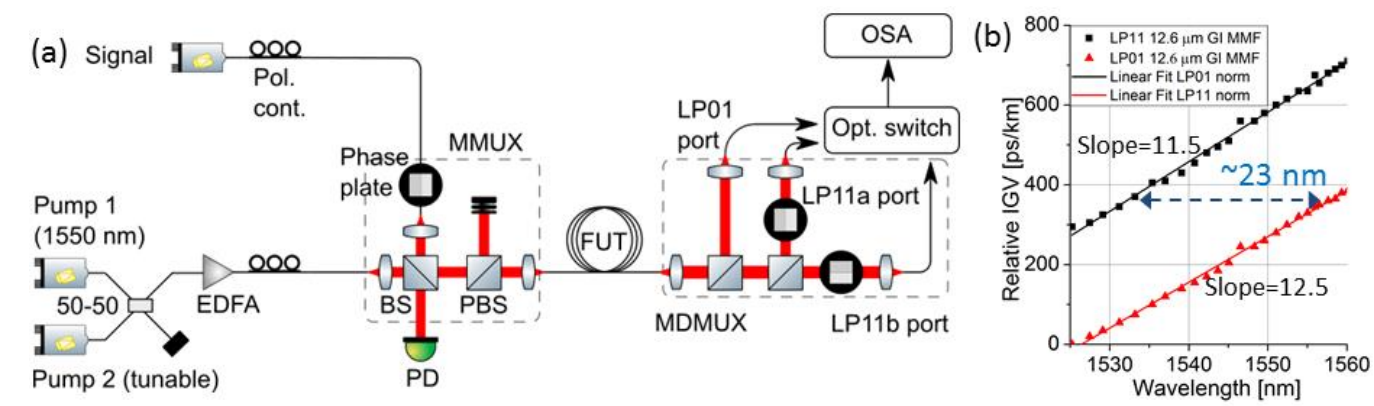

Fig. 2: (a) Intermodal BS FWM experimental set-up using the fabricated dispersion engineered GI MMF with core radius of $12.6 \mu \mathrm{m}$ and corresponding measured relative IGV curves for the $L P_{01}$ and $L P_{11}$ modes (b).

index profile shown in Fig. 1(b).

Several GI MMFs were then fabricated from a single preform with different core radii, ranging between $9.6 \mu \mathrm{m}$ and $12.6 \mu \mathrm{m}$. The core was made of highly $\mathrm{GeO}_{2}$-doped $\mathrm{SiO}_{2}$ and the cladding of pure $\mathrm{SiO}_{2}$, similar to conventional single-mode silica-based highly nonlinear fibres ${ }^{7}$. The index profile in the core has a peak index difference of $2.0 \%$ relative to the cladding and a fitted alpha-power of 2.1, see Fig.1 (b). Thanks to the mature material technology, the measured attenuation for the $\mathrm{LP}_{01}$ mode was as low as $0.3 \mathrm{~dB} / \mathrm{km}$. Similar losses are expected for the $\mathrm{LP}_{11}$ mode.

Simulations, obtained by solving the multi-mode generalized nonlinear Schrödinger equations, highlight that by increasing the core diameter of the fibre, the wavelength separation between the phase matched waves (pumps and signal-idler

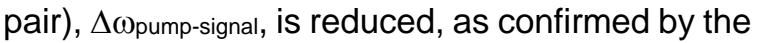
measured inverse group velocity (IGV) curves of the first two modes, see Fig. 1(c), while maintaining relatively constant $\Delta \omega \mathrm{pp}$ bandwidths (as long as the higher order dispersion values of the modes do not start to play an important role).

\section{Experimental Set-up}

The experimental set-up is shown in Fig. 2(a). Three continuous-wave laser sources were used to generate co-polarized pump and signal waves at the fibre input. The pumps, $P_{1}$ and $P_{2}$, were launched into the fundamental mode and the wavelength of $P_{1}$ was kept constant at $1550 \mathrm{~nm}$, while that of $\mathrm{P}_{2}$ was varied. The signal wave was launched into the $L P_{11}$ mode, using a free-space phase plate (PP) based mode-multiplexer (MMUX), see Fig. 2(a). The signal wavelength was tuned to obtain the largest bandwidth. The pump powers into the fibre were typically $23.5 \mathrm{dBm}$ total, while the signal was about 8.5 $\mathrm{dBm}$. While the $100 \mathrm{~m}$ long GI MMF supports many modes, we only focused on the LP 01 and $\mathrm{LP}_{11}$ mode groups. To guarantee the right modal excitation the GI MMF was spliced to a two-mode fibre. At the GI MMF output, the different spatial modes were extracted using a modedemultiplexer (MDMUX) based on the same freespace technology as the MMUX followed by single-mode fibres. Note that even though the MMUX excites only one of the $L P_{11}$ modes, both modes $\mathrm{LP}_{11 \mathrm{a}}$ and $\mathrm{LP}_{11 \mathrm{~b}}$ become completely mixed after propagation along the GI MMF and, thus, having two orthogonally orientated PPs in the MDMUX is essential in order to collect the total power in the $\mathrm{LP}_{11}$ mode group. An optical switch enabled the selection and independent measurement of each of the MDMUX output ports on an optical spectrum analyser (OSA). Both the MMUX and MDMUX gave typical mode extinction ratios of about $20 \mathrm{~dB}$.

We measured the IGV curves of the supported modes of all the fabricated GI MMFs via a timeof-flight measurement. Figure 2(b) shows an example of the relative IGV for the first two modes of the GI MMF with a core radius of 12.6 $\mu \mathrm{m}$. The horizontal arrow in the figure illustrates the conditions for phase matching between the two modes (assuming $\Delta \omega_{p p}=0$ ), corresponding to a pump-signal wavelength separation of about 23 $\mathrm{nm}$.

\section{Simulated and Experimental Results}

By applying a linear fit to the measured IGV curves, we simulated the BSr and BSb FWM process in terms of $\Delta \omega p$ pandwidth for two slightly different signal wavelengths. Figure 3 (a) and (b) show the normalized simulated conversion efficiency (CE): $-3 \mathrm{~dB}$ half-bandwidths of almost $9 \mathrm{~nm}$ and $18 \mathrm{~nm}$ can be obtained for the phase matched BSr process, but only $0.5 \mathrm{~nm}$ for the BSb case. The maximum CE is about $-32 \mathrm{~dB}$. Two different signal wavelengths detuned by about $0.6 \mathrm{~nm}$ were considered. As previously observed $^{1}$, by slightly tuning the signal wavelength from the theoretical phase matching point it is possible to enlarge the $-3 \mathrm{~dB}$ bandwidth despite the presence of a small depression in the middle of the band.

We then performed the corresponding 

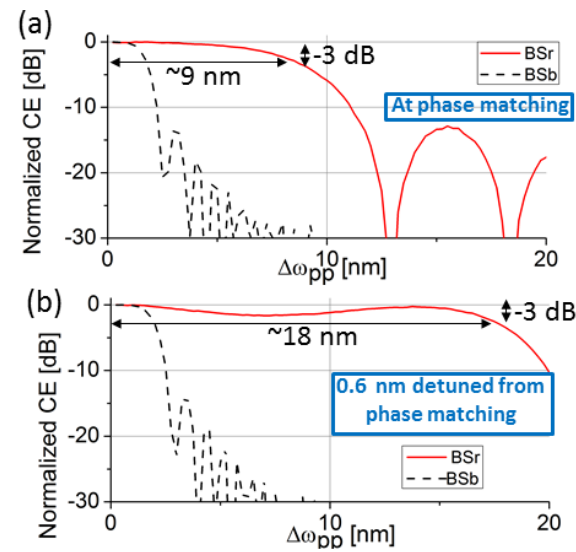

Fig. 3: Simulated normalized conversion efficiency of the BSr (solid line) and the BSb (dashed line) processes for the $12.6 \mu \mathrm{m}$ core radius GI MMF for two signal wavelengths separated by $0.6 \mathrm{~nm}$.

experiments. Figure 4(a) shows typical intermodal FWM spectra obtained at the recombined $L P_{11}$ MDMUX output port when the $12.6 \mu \mathrm{m}$ core radius $\mathrm{Gl}$ MMF is used with a $\Delta \omega \mathrm{pp}$ of $0.5 \mathrm{~nm}$ and $3 \mathrm{~nm}$, respectively, and $\Delta \omega_{\text {pump-signal }}$ of about $23 \mathrm{~nm}$. The signal wavelength was chosen to give the widest bandwidth. As expected from the simulations, when $\Delta \omega_{\mathrm{pp}}$ increases from $0.5 \mathrm{~nm}$ to $3 \mathrm{~nm}$ the phase matched BSr idler remains fairly constant, while the non-phase matched BSb idler vanishes for $\Delta \omega_{\mathrm{pp}}=3 \mathrm{~nm}$. The optical signal to noise ratios of the BSr idlers are more than $20 \mathrm{~dB}$ for both cases, but the corresponding measured conversion efficiencies are quite low, about $-38 \mathrm{~dB}$. This is due to the relatively large core radius used and could potentially be improved significantly by more complex fibre designs with a smaller core radius or by moving to highly nonlinear materials. We then studied the CE of the BSb and BSr idlers depending on the $\Delta \omega_{\mathrm{pp}}$ detuning. The normalized CE curves are shown in Fig. 4(b), demonstrating a $-3 \mathrm{~dB}$ half-bandwidth of about $7 \mathrm{~nm}$ for the $\mathrm{BSr}$ idler, while the bandwidth is as small as $0.5 \mathrm{~nm}$ for the BSb idler. This clearly highlights that it is possible to selectively optimize the strength of the desired nonlinear process, BSr versus BSb, by properly engineering the modes of the fibre. We attribute the discrepancy between the simulated and experimental bandwidths to higher order dispersion terms that were not included in the simulations and to modal dispersion fluctuations along the fabricated fibre ${ }^{8}$. Similar measurements, not shown here, were performed for a GI MMF with a smaller core radius of $12 \mu \mathrm{m}$. In this case, phase matched BS can be achieved for a larger $\Delta \omega_{\text {pump-signal }}$ of $33.8 \mathrm{~nm}$. For this fibre the measured half-bandwidth was reduced to 4 $\mathrm{nm}$. We believe that this is due to the fact that as
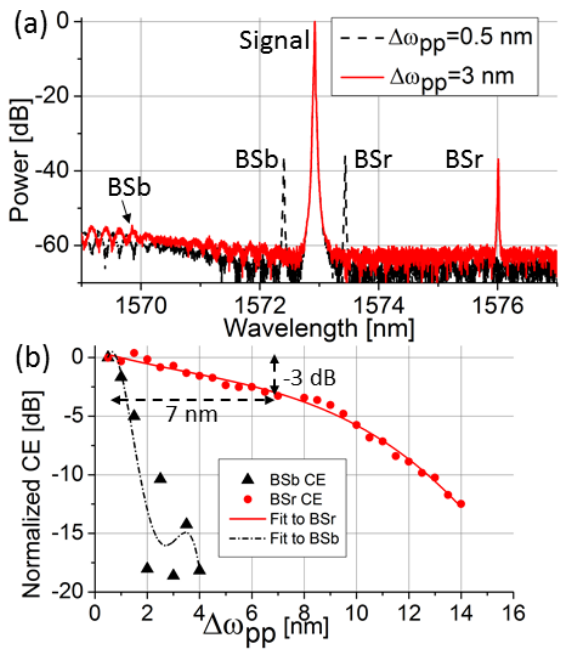

Fig. 4: Measured spectral traces for $\Delta \omega_{\mathrm{pp}}$ of $0.5 \mathrm{~nm}$ and $3 \mathrm{~nm}$, respectively, (a) and normalized conversion efficiency of the BSr (circles) and the BSb (triangle) processes for the $12.6 \mu \mathrm{m}$ core radius GI MMF (b).

the $\Delta \omega$ pump-signal increases, higher order modal dispersion terms start playing a more critical role in the FWM process, especially if stochastic variations of the key fibre parameters (e. g. core radius) occur along its length.

\section{Conclusions}

We have reported the design, fabrication and characterisation of dispersion engineered graded index multi-mode fibres offering broadband operation for the inter-modal Bragg scattering FWM process. We measured bandwidths of up to $7 \mathrm{~nm}$ in the fabricated dispersion-engineered fibres, a much broader value than obtained in previously reported work.

\section{References}

[1] F. Parmigiani, et al., "All-optical mode and wavelength converter based on parametric processes in a threemode fiber," Opt. Express, Vol. 25, p. 33602 (2017).

[2] F. Parmigiani, et al.," Polarization Insensitive Wavelength Conversion in a Few Mode Fibre" W.2.F.2, ECOC 2017.

[3] M. Esmaeelpour, et al., "Power fluctuations of intermodal four-wave mixing in few-mode fibers," J. Lightwave Technol., Vol. 35, no. 12, p. 2429 (2017).

[4] J. Demas, et al., "High-power, wavelength-tunable NIR all-fiber lasers via intermodal four-wave mixing," JTh5A.8, CLEO US (2017).

[5] A. Bendahmane, et al., "Seeded intermodal four-wave mixing in a highly multimode fiber," J. Opt. Soc. Am. B, Vol. 35, p. 295 (2018).

[6] C. J. McKinstrie, et al., "Translation of quantum states by four-wave mixing," Opt. Express, Vol. 13, p. 9131 (2005).

[7] M. Hirano, et al., "Silica-based Highly Nonlinear Fiber Advances," Tu2E.4, OFC 2016.

[8] M. Guasoni, et al., "Intermodal four-wave mixing and parameteric amplification in kilometer-long multimode fibers," J. Lightwave Technol., Vol. 35, p. 5296 (2017). 